\title{
Persistence and Nonpersistence of a Food Chain Model with Stochastic Perturbation
}

\author{
Haihong Li, ${ }^{1,2}$ Fuzhong Cong, ${ }^{1,2}$ Daqing Jiang, ${ }^{3}$ and Hongtu Hua ${ }^{2}$ \\ ${ }^{1}$ School of Mathematics, Jilin University, Changchun, Jilin 130024, China \\ ${ }^{2}$ Department of Basic Courses, Air Force Aviation University, Changchun, Jilin 130022, China \\ ${ }^{3}$ School of Mathematics, Northeast Normal University, Changchun, Jilin 130024, China \\ Correspondence should be addressed to Fuzhong Cong; congfz67@126.com
}

Received 3 June 2013; Accepted 21 September 2013

Academic Editor: Mark McKibben

Copyright (C) 2013 Haihong Li et al. This is an open access article distributed under the Creative Commons Attribution License, which permits unrestricted use, distribution, and reproduction in any medium, provided the original work is properly cited.

We analyze a three species predator-prey chain model with stochastic perturbation. First, we show that this system has a unique positive solution and its $p$ th moment is bounded. Then, we deduce conditions that the system is persistent in time average. After that, conditions for the system going to be extinction in probability are established. At last, numerical simulations are carried out to support our results.

\section{Introduction}

Recently, the dynamical relationship between predator-prey has been one of the dominant themes in both ecology and mathematical ecology due to its universal importance. Especially, the predator-prey chain model is the typical representative. Thereby it significantly changed the biology, the understanding of the existence, and development of the basic law and has made the model become a research hot spot. One of the most famous models for population dynamics is the Lotka-Volterra predator-prey system which has received plenty of attention and has been studied extensively; see [14]. Specially persistence and extinction of this model are interesting topics.

The three species predator-prey chain model is described as follows:

$$
\begin{aligned}
& \dot{x}_{1}(t)=x_{1}(t)\left(a_{1}-b_{11} x_{1}(t)-b_{12} x_{2}(t)\right), \\
& \dot{x}_{2}(t)=x_{2}(t)\left(-a_{2}+b_{21} x_{1}(t)-b_{22} x_{2}(t)-b_{23} x_{3}(t)\right), \\
& \dot{x}_{3}(t)=x_{3}(t)\left(-a_{3}+b_{32} x_{2}(t)-b_{33} x_{3}(t)\right),
\end{aligned}
$$

where $x_{i}(t)(i=1,2,3)$ denotes the population densities of the species at time $t$. The parameters $a_{1}, a_{2}, a_{3}, b_{i i}(i=1,2,3)$ are positive constants that stand for intrinsic growth rate, predator death rate of the second species, predator death rate of the third species, coefficient of internal competition, respectively. $b_{21}, b_{32}$ represent saturated rate of the second and the third predator and $b_{12}, b_{23}$ represent the decrement rate of predator to prey.

System (1) describes a three species predator-prey chain model in which the latter preys on the former. From a biological viewpoint, we not only require the positive solution of the system but also require its unexploded property in any finite time and stability.

We know that the global asymptotic stability of a positive equilibrium $x^{*}=\left(x_{1}^{*}, x_{2}^{*}, x_{3}^{*}\right)$ holds and is global stability if the following condition holds:

$$
a_{1}-\frac{b_{11}}{b_{21}} a_{2}-\frac{b_{11} b_{22}+b_{12} b_{21}}{b_{21} b_{32}} a_{3}>0
$$

which could refer to [5]. However, population dynamics in the real world is inevitably affected by environmental noise (see, e.g., $[6,7])$. Parameters involved in the system are not absolute constants, they always fluctuate around some average values. The deterministic models assume that parameters in the systems are deterministic irrespective of environmental fluctuations which impose some limitations in mathematical modeling of ecological systems. So we cannot omit the influence of the noise on the system. Recently many authors have discussed population systems subject to white 
noise (see, e.g., [8-15]). May (see, e.g., [16]) pointed out that due to continuous fluctuation in the environment, the birth rates, death rates, saturated rate, competition coefficients, and all other parameters involved in the model exhibit random fluctuation to some extent, and as a result the equilibrium population distribution never attains a steady value but fluctuates randomly around some average value. Sometimes, large amplitude fluctuation in population will lead to the extinction of certain species, which does not happen in deterministic models.

Therefore, Lotka-Volterra predator-prey chain models in random environments are becoming more and more popular. Ji et al. $[14,15]$ investigated the asymptotic behavior of the stochastic predator-prey system with perturbation. Liu and Chen introduced periodic constant impulsive immigration of predator into predator-prey system and gave conditions for the system to be extinct and permanence. Polansky [17] and Barra et al. [18] have given some special systems of their invariant distribution. After that, Gard [5] analysed that under some conditions the stochastic food chain model exists an invariant distribution. However, seldom people study the persistent and nonpersistent of the food chain model with stochastic perturbation.

In this paper, we introduce the white noise into the intrinsic growth rate of system (1), and suppose $a_{i} \rightarrow a_{i}+$ $\sigma_{i} \dot{B}_{i}(t)(i=1,2,3)$; then we obtain the following stochastic system:

$$
\begin{aligned}
\dot{x}_{1}(t)= & x_{1}(t)\left(a_{1}-b_{11} x_{1}(t)-b_{12} x_{2}(t)\right)+\sigma_{1} x_{1}(t) \dot{B}_{1}(t), \\
\dot{x}_{2}(t)= & x_{2}(t)\left(-a_{2}+b_{21} x_{1}(t)-b_{22} x_{2}(t)-b_{23} x_{3}(t)\right) \\
& -\sigma_{2} x_{2}(t) \dot{B}_{2}(t), \\
\dot{x}_{3}(t)= & x_{3}(t)\left(-a_{3}+b_{32} x_{2}(t)-b_{33} x_{3}(t)\right)-\sigma_{3} x_{3}(t) \dot{B}_{3}(t),
\end{aligned}
$$

where $B_{i}(t)(i=1,2,3)$ are independent white noises with $B_{i}(0)=0, \sigma_{i}^{2}>0(i=1,2,3)$ representing the intensities of the noise.

The aim of this paper is to discuss the long time behavior of system (3). We have mentioned that $x^{*}=\left(x_{1}^{*}, x_{2}^{*}, x_{3}^{*}\right)$ is the positive equilibrium of system (1). But, when it suffers stochastic perturbations, there is no positive equilibrium. Hence, it is impossible that the solution of system (3) will tend to a fixed point. In this paper, we show that system (3) is persistent in time average. Furthermore, under certain conditions, we prove that the population of system (3) will die out in probability which will not happen in deterministic system and could reveal that large white noise may lead to extinction.

The rest of this paper is organized as follows. In Section 2, we show that there is a unique nonnegative solution of system (3), and its pth moment is bounded. In Section 3, we show that system (3) is persistent in time average. While in Section 4, we consider three situations when the population of the system will be extinction. In Section 5, numerical simulations are carried out to support our results.
Throughout this paper, unless otherwise specified, let $\left(\Omega,\left\{\mathscr{F}_{t}\right\}_{t \geq 0}, P\right)$ be a complete probability space with a filtration $\left\{\mathscr{F}_{t}\right\}_{t \geq 0}$ satisfying the usual conditions (i.e., it is right continuous and $\mathscr{F}_{0}$ contains all $P$-null sets). Let $R_{+}^{3}$ denote the positive cone of $R^{3}$; namely, $R_{+}^{3}=\left\{x \in R^{3}: x_{i}>0,1 \leq i \leq 3\right\}$, $\bar{R}_{+}^{3}=\left\{x \in R^{3}: x_{i} \geq 0,1 \leq i \leq 3\right\}$.

\section{Existence and Uniqueness of the Nonnegative Solution}

To investigate the dynamical behavior, the first concern thing is whether the solution is global existence. Moreover, for a population model, whether the solution is nonnegative is also considered. Hence, in this section, we show that the solution of system (3) is global and nonnegative. As we have known, in order for a stochastic differential equation to have a unique global (i.e., no explosion at a finite time) solution with any given initial value, the coefficients of the equation are generally required to satisfy the linear growth condition and local Lipschitz condition (see, e.g., [19]). It is easy to see that the coefficients of system (3) are locally Lipschitz continuous, so system (3) has a local solution. By Lyapunov analysis method, we show the global existence of this solution.

Theorem 1. For any given initial value $x(0)=x_{0} \in R_{+}^{3}$, system (3) has a unique global positive solution $x(t)=\left(x_{1}(t), x_{2}(t)\right.$, $\left.x_{3}(t)\right)$ for all $t \geq 0$ with probability one.

Proof. It is clear that the coefficients of system (3) are locally Lipschitz continuous for the given initial value $x(0)=x_{0} \in$ $R_{+}^{3}$. So there is a unique local solution $x(t)$ on $t \in\left[0, \tau_{e}\right)$, where $\tau_{e}$ is the explosion time (see, e.g., [19]). To show that this solution is global, we need to show that $\tau_{e}=\infty$ a.s. Let $m_{0} \geq 1$ be sufficiently large so that each component of $x_{0}$ all lies within the interval $\left[1 / m_{0}, m_{0}\right]$. For each integer $m \geq m_{0}$, define the stopping time:

$$
\begin{aligned}
\tau_{m}=\inf \left\{t \in\left[0, \tau_{e}\right):\right. & \min \left\{x_{1}(t), x_{2}(t), x_{3}(t)\right\} \leq \frac{1}{m} \\
& \text { or } \left.\max \left\{x_{1}(t), x_{2}(t), x_{3}(t)\right\} \geq m\right\} .
\end{aligned}
$$

Throughout this paper, we set $\inf \emptyset=\infty$ (as usual $\emptyset$ denotes the empty set). Clearly, $\tau_{m}$ is increasing as $m \rightarrow \infty$. Set $\tau_{\infty}=$ $\lim _{m \rightarrow \infty} \tau_{m}$; then $\tau_{\infty} \leq \tau_{e}$ a.s. If we can show that $\tau_{\infty}=\infty$ a.s., then $\tau_{e}=\infty$ and $\left(x_{1}(t), x_{2}(t), x_{3}(t)\right) \in R_{+}^{3}$ a.s. for all $t \geq 0$. In other words, to complete the proof all we need to show is that $\tau_{\infty}=\infty$ a.s. For if this statement is false, then there is a pair of constants $T>0$ and $\epsilon \in(0,1)$ such that

$$
P\left\{\tau_{\infty} \leq T\right\}>\epsilon
$$

Hence there is an integer $m_{1} \geq m_{0}$ such that

$$
P\left\{\tau_{m} \leq T\right\} \geq \epsilon \quad \forall m \geq m_{1} .
$$


Define a $C^{2}$-function $V: R_{+}^{3} \rightarrow \bar{R}_{+}$by

$$
\begin{aligned}
& V\left(x_{1}, x_{2}, x_{3}\right) \\
& =b_{32}\left[b_{21}\left(x_{1}-1-\log x_{1}\right)+b_{12}\left(x_{2}-1-\log x_{2}\right)\right] \\
& \quad+b_{23} b_{12}\left(x_{3}-1-\log x_{3}\right) ;
\end{aligned}
$$

the nonnegativity of this function can be seen from $u-1-$ $(1 / 2) \log u \geq 0$, for all $u>0$. Using Itô's formula, we get

$$
\begin{aligned}
d V:= & L V d t+b_{32} b_{21} \sigma_{1}\left(x_{1}-1\right) d B_{1}(t) \\
& +b_{32} b_{12} \sigma_{2}\left(x_{2}-1\right) d B_{2}(t) \\
& +b_{23} b_{12} \sigma_{3}\left(x_{3}-1\right) d B_{3}(t),
\end{aligned}
$$

where

$$
\begin{aligned}
L V= & b_{32} b_{21}\left(x_{1}-1\right)\left(a_{1}-b_{11} x_{1}-b_{12} x_{2}\right) \\
& +b_{32} b_{12}\left(x_{2}-1\right)\left(-a_{2}+b_{21} x_{1}-b_{22} x_{2}-b_{23} x_{3}\right) \\
& +b_{23} b_{12}\left(x_{3}-1\right)\left(-a_{3}+b_{32} x_{2}-b_{33} x_{3}\right) \\
& +b_{32} b_{21} \frac{\sigma_{1}^{2}}{2}+b_{32} b_{12} \frac{\sigma_{2}^{2}}{2}+b_{23} b_{12} \frac{\sigma_{1}^{2}}{2} \\
= & b_{32} b_{21}\left(-a_{1}+\frac{\sigma_{1}^{2}}{2}\right)+b_{32} b_{12}\left(a_{2}+\frac{\sigma_{2}^{2}}{2}\right) \\
& +b_{23} b_{12}\left(a_{3}+\frac{\sigma_{3}^{2}}{2}\right) \\
& +\left(b_{32} b_{21} a_{1}+b_{32} b_{21} b_{11}-b_{32} b_{12} b_{21}\right) x_{1} \\
& +\left(b_{32} b_{21} b_{12}+b_{32} b_{12} b_{22}-b_{23} b_{12} b_{32}-b_{32} b_{12} a_{2}\right) x_{2} \\
& +\left(b_{32} b_{12} b_{23}+b_{23} b_{12} b_{33}-b_{23} b_{12} a_{3}\right) x_{3} \\
& -b_{32} b_{21} b_{11} x_{1}^{2}-b_{32} b_{12} b_{22} x_{2}^{2}-b_{23} b_{12} b_{33} x_{3}^{2} \leq \widehat{M}
\end{aligned}
$$

$$
\begin{aligned}
& +\int_{0}^{\tau_{m} \wedge T} b_{32} b_{12} \sigma_{2}\left(x_{2}-1\right) d B_{2}(t) \\
& +\int_{0}^{\tau_{m} \wedge T} b_{23} b_{12} \sigma_{3}\left(x_{3}-1\right) d B_{3}(t),
\end{aligned}
$$

which implies that

$$
E\left[V\left(x\left(\tau_{m} \wedge T\right)\right)\right] \leq V\left(x_{0}\right)+\widehat{M} T .
$$

Set $\Omega_{m}=\left\{\tau_{m} \leq T\right\}$ for $m \geq m_{1}$. By (6), we know $P\left(\Omega_{m}\right) \geq \epsilon$. Notice that for every $\omega \in \Omega_{m}$, there is at least one of $x_{i}\left(\tau_{m}, \omega\right)$ equals either $m$ or $1 / m$; then

$$
V\left(x\left(\tau_{m}\right)\right) \geq(m-1-\log m) \wedge\left(m^{-1}-1+\log m\right) .
$$

It then follows from (11) that

$$
\begin{aligned}
V\left(x_{0}\right) & +\widehat{M} T \\
& \geq E\left[1_{\Omega_{m}(\omega)} V\left(x\left(\tau_{m}\right)\right)\right] \\
& \geq \epsilon(m-1-\log m) \wedge\left(m^{-1}-1+\log m\right),
\end{aligned}
$$

where $1_{\Omega_{m}(\omega)}$ is the indicator function of $\Omega_{m}$. Letting $m \rightarrow$ $\infty$ leads to the contradiction that $\infty>V\left(x_{0}\right)+\widehat{M} T=\infty$. So we must have $\tau_{\infty}=\infty$ a.s.

Theorem 2. Let $x(t)=\left(x_{1}(t), x_{2}(t)\right.$, and let $\left.x_{3}(t)\right)$ be the solution of system (3) with any given initial value $x(0)=x_{0} \epsilon$ $R_{+}^{3}$, then there exists a positive constant $K(p)$ such that

$$
\begin{array}{r}
E\left[\left(b_{32} b_{21} x_{1}(t)+b_{32} b_{12} x_{2}(t)+b_{23} b_{12} x_{3}(t)\right)^{p}\right] \leq K(p) \\
\forall t \in(0, \infty), \quad p>1 .
\end{array}
$$

Proof. Let $y(t)=b_{32} b_{21} x_{1}(t)+b_{32} b_{12} x_{2}(t)+b_{23} b_{12} x_{3}(t)$; then

$$
\begin{aligned}
d y(t)= & \left(a_{1} b_{21} b_{32} x_{1}-a_{2} b_{12} b_{32} x_{2}-a_{3} b_{12} b_{23} x_{3}\right. \\
& \left.-b_{11} b_{21} b_{32} x_{1}^{2}-b_{12} b_{22} b_{32} x_{2}^{2}-b_{12} b_{23} b_{33} x_{3}^{2}\right) d t \\
& +\sigma_{1} b_{21} b_{32} x_{1} d B_{1}(t)+\sigma_{2} b_{12} b_{32} x_{2} d B_{2}(t) \\
& +\sigma_{3} b_{12} b_{23} x_{3} d B_{3}(t)
\end{aligned}
$$

and so

$$
\begin{gathered}
d y^{p}=p y^{p-1}\left(a_{1} b_{21} b_{32} x_{1}-a_{2} b_{12} b_{32} x_{2}-a_{3} b_{12} b_{23} x_{3}\right. \\
\quad-b_{11} b_{21} b_{32} x_{1}^{2}-b_{12} b_{22} b_{32} x_{2}^{2} \\
\left.\quad-b_{12} b_{23} b_{33} x_{3}^{2}\right) d t \\
+p y^{p-1}\left(\sigma_{1} b_{21} b_{32} x_{1} d B_{1}(t)+\sigma_{2} b_{12} b_{32} x_{2} d B_{2}(t)\right. \\
\left.\quad+\sigma_{3} b_{12} b_{23} x_{3} d B_{3}(t)\right) \\
+\frac{1}{2} p(p-1) y^{p-2} \\
\quad \times\left(\sigma_{1}^{2} b_{21}^{2} b_{32}^{2} x_{1}^{2}+\sigma_{2}^{2} b_{12}^{2} b_{32}^{2} x_{2}^{2}+\sigma_{3}^{2} b_{12}^{2} b_{23}^{2} x_{3}^{2}\right) d t .
\end{gathered}
$$

Note that

$$
\begin{aligned}
b_{11} b_{21} b_{32} x_{1}^{2}+b_{12} b_{22} b_{32} x_{2}^{2}+b_{12} b_{23} b_{33} x_{3}^{2} \\
\geq \frac{\min \left\{b_{11}, b_{22}, b_{33}\right\}}{b_{32} b_{21}+b_{32} b_{12}+b_{23} b_{12}} \\
\quad \times\left(b_{32} b_{21} x_{1}+b_{32} b_{12} x_{2}+b_{23} b_{12} x_{3}\right)^{2} \\
=\frac{\min \left\{b_{11}, b_{22}, b_{33}\right\}}{b_{32} b_{21}+b_{32} b_{12}+b_{23} b_{12}} y^{2} .
\end{aligned}
$$


Then

$$
\begin{aligned}
d y^{p}= & p y^{p-1}\left(a_{1} y-\frac{\min \left\{b_{11}, b_{22}, b_{33}\right\}}{b_{32} b_{21}+b_{32} b_{12}+b_{23} b_{12}} y^{2}\right) d t \\
& +\frac{1}{2} p(p-1) y^{p-2} \max \left\{\sigma_{1}^{2}, \sigma_{2}^{2}, \sigma_{3}^{2}\right\} y^{2} d t \\
& +p y^{p-1}\left(\sigma_{1} b_{21} b_{32} x_{1} d B_{1}(t)+\sigma_{2} b_{12} b_{32} x_{2} d B_{2}(t)\right. \\
& \left.+\sigma_{3} b_{12} b_{23} x_{3} d B_{3}(t)\right) \\
\leq & {\left[p\left(a_{1}+\frac{p}{2} \max \left\{\sigma_{1}^{2}, \sigma_{2}^{2}, \sigma_{3}^{2}\right\}\right) y^{p}\right.} \\
& \left.\quad-\frac{p \min \left\{b_{11}, b_{22}, b_{33}\right\}}{b_{32} b_{21}+b_{32} b_{12}+b_{23} b_{12}} y^{p+1}\right] d t \\
+ & p y^{p-1}\left(\sigma_{1} b_{21} b_{32} x_{1} d B_{1}(t)+\sigma_{2} b_{12} b_{32} x_{2} d B_{2}(t)\right. \\
& \left.+\sigma_{3} b_{12} b_{23} x_{3} d B_{3}(t)\right) .
\end{aligned}
$$

Hence

$$
\begin{aligned}
& \frac{d E\left[y^{p}(t)\right]}{d t} \\
& \quad \leq p\left(a_{1}+p \frac{\max \left\{\sigma_{1}^{2}, \sigma_{2}^{2}, \sigma_{3}^{2}\right\}}{2}\right) E\left[y^{p}(t)\right] \\
& \quad-p \frac{\min \left\{b_{11}, b_{22}, b_{33}\right\}}{b_{32} b_{21}+b_{32} b_{12}+b_{23} b_{12}} E\left[y^{p+1}(t)\right] \\
& \leq p\left(a_{1}+p \frac{\max \left\{\sigma_{1}^{2}, \sigma_{2}^{2}, \sigma_{3}^{2}\right\}}{2}\right) E\left[y^{p}(t)\right] \\
& \quad-p \frac{\min \left\{b_{11}, b_{22}, b_{33}\right\}}{b_{32} b_{21}+b_{32} b_{12}+b_{23} b_{12}} E\left[y^{p}(t)\right]^{(p+1) / p} .
\end{aligned}
$$

Therefore, by comparison theorem, we get

$$
\begin{aligned}
& \limsup _{t \rightarrow \infty} E\left[y^{p}(t)\right] \\
& \leq\left[\frac{\left(a_{1}+p \max \left\{\sigma_{1}^{2}, \sigma_{2}^{2}, \sigma_{3}^{2}\right\}\right)\left(b_{32} b_{21}+b_{32} b_{12}+b_{23} b_{12}\right)}{\min \left\{b_{11}, b_{22}, b_{33}\right\}}\right]^{p} .
\end{aligned}
$$

Besides, note that $E\left[y^{p}(t)\right]$ is continuous; then there is a positive constant $K(p)$ such that

$$
E\left[y^{p}(t)\right] \leq K(p), \quad \forall t \in[0, \infty) .
$$

\section{Persistent in Time Average}

There is no equilibrium of system (3). Hence we cannot show the permanence of the system by proving the stability of the positive equilibrium as the deterministic system. In this section we first show that this system is persistent in mean. Before we give the result, we should do some prepared work.
L. S. Chen and J. Chen in [20] proposed the definition of persistence in mean for the deterministic system. Here, we also use this definition for the stochastic system.

Definition 3. System (3) is said to be persistent in mean, if

$$
\liminf _{t \rightarrow \infty} \frac{1}{t} \int_{0}^{t} x_{3}(s) d s>0 \text {, a.s. }
$$

Lemma 4 (see [21, Lemma 17]). Let $f \in C([0,+\infty) \times$ $\Omega,(0,+\infty))$ and $F \in C([0,+\infty) \times \Omega, R)$. If there exist positive constants $\lambda_{0}, \lambda$, such that

$$
\log f(t) \geq \lambda t-\lambda_{0} \int_{0}^{t} f(s) d s+F(t), \quad t \geq 0 \text { a.s., }
$$

and $\lim _{t \rightarrow \infty}(F(t) / t)=0$ a.s., then

$$
\liminf _{t \rightarrow \infty} \frac{1}{t} \int_{0}^{t} f(s) d s \geq \frac{\lambda}{\lambda_{0}}, \text { a.s. }
$$

From Lemma 4, it is easy to see that we could get Lemmas 5 and 6 with the same method.

Lemma 5. Let $f \in C([0,+\infty) \times \Omega,(0,+\infty))$ and $F \in C([0$, $+\infty) \times \Omega, R)$. If there exist positive constants $\lambda_{0}$, $\lambda$, such that

$$
\log f(t) \leq \lambda t-\lambda_{0} \int_{0}^{t} f(s) d s+F(t), \quad t \geq 0 \text { a.s. }
$$

and $\lim _{t \rightarrow \infty}(F(t) / t)=0$ a.s., then

$$
\limsup _{t \rightarrow \infty} \frac{1}{t} \int_{0}^{t} f(s) d s \leq \frac{\lambda}{\lambda_{0}}, \text { a.s. }
$$

Lemma 6. Let $f \in C([0,+\infty) \times \Omega,(0,+\infty))$ and $F \in C([0$, $+\infty) \times \Omega, R)$. If there exist positive constants $\lambda_{0}, \lambda$, such that

$$
\log f(t)=\lambda t-\lambda_{0} \int_{0}^{t} f(s) d s+F(t), \quad t \geq 0 \text { a.s., }
$$

and $\lim _{t \rightarrow \infty}(F(t) / t)=0$ a.s., then

$$
\lim _{t \rightarrow \infty} \frac{1}{t} \int_{0}^{t} f(s) d s=\frac{\lambda}{\lambda_{0}}, \text { a.s. }
$$

From the stochastic comparison theorem [11], it is easy to get the following result.

Lemma 7. Let $x(t) \in R_{+}^{3}$ be a solution of system (3) with $x(0)=\left(x_{1}(0), x_{2}(0), x_{3}(0)\right)$. Then one has

$$
x(t) \leq \Phi(t)
$$

that is,

$$
x_{i}(t) \leq \Phi_{i}(t), \quad i=1,2,3,
$$

where

$$
\Phi(t)=\left(\Phi_{1}(t), \Phi_{2}(t), \Phi_{3}(t)\right)^{\top},
$$


$\Phi_{i}(t)$ is solutions of the following stochastic differential equations:

$$
\begin{aligned}
d \Phi_{1}(t)= & \Phi_{1}(t)\left(a_{1}-b_{11} \Phi_{1}(t)\right) d t \\
& +\sigma_{1} \Phi_{1}(t) d B_{1}(t), \quad \Phi_{1}(0)=x_{1}(0), \\
d \Phi_{2}(t)= & \Phi_{2}(t)\left(-a_{2}+b_{21} \Phi_{1}(t)-b_{22} \Phi_{2}(t)\right) d t \\
& -\sigma_{2} \Phi_{2}(t) d B_{2}(t), \quad \Phi_{2}(0)=x_{2}(0), \\
d \Phi_{3}(t)= & \Phi_{3}(t)\left(-a_{3}+b_{32} \Phi_{2}(t)-b_{33} \Phi_{3}(t)\right) d t \\
& -\sigma_{3} \Phi_{3}(t) d B_{3}(t), \quad \Phi_{3}(0)=x_{3}(0) .
\end{aligned}
$$

Assumption 8. Consider

$$
\begin{gathered}
r_{1}-\frac{b_{11}}{b_{21}} r_{2}-\frac{b_{11} b_{22}+b_{12} b_{21}}{b_{21} b_{32}} r_{3}>0, \\
r_{1}=a_{1}-\frac{\sigma_{1}^{2}}{2}>0, \quad r_{i}=a_{i}+\frac{\sigma_{i}^{2}}{2} \quad i=2,3 .
\end{gathered}
$$

Lemma 9. If Assumption 8 is satisfied, the solution $\Phi(t)$ of system (32) with any initial value $\Phi(0) \in R_{+}^{3}$ has the following property:

$$
\lim _{t \rightarrow \infty} \frac{\log \Phi_{i}(t)}{t}=0, \quad \lim _{t \rightarrow \infty} \frac{1}{t} \int_{0}^{t} \Phi_{i}(s) d s=M_{i}, \text { a.s. }
$$

where

$$
\begin{aligned}
& M_{1}=\frac{r_{1}}{b_{11}}, \quad M_{2}=\frac{r_{1} b_{21}-r_{2} b_{11}}{b_{11}}, \\
& M_{3}=\frac{r_{1} b_{21} b_{32}-r_{2} b_{11} b_{32}-r_{3} b_{11} b_{22}}{b_{11} b_{22} b_{33}} .
\end{aligned}
$$

Proof. From the result in [14] and Assumption 8 being satisfied, we know

$$
\begin{gathered}
\lim _{t \rightarrow \infty} \frac{\log \Phi_{1}(t)}{t}=0, \\
\liminf _{t \rightarrow \infty} \frac{1}{t} \int_{0}^{t} \Phi_{1}(s) d s=\frac{a_{1}-\sigma_{1}^{2} / 2}{b_{11}}=\frac{r_{1}}{b_{11}},
\end{gathered}
$$

Besides, according to Itô's formula, the second population of system (32) is changed into

$$
d \log \Phi_{2}(t)=\left(-r_{2}+b_{21} \Phi_{1}(t)-b_{22} \Phi_{2}(t)\right) d t-\sigma_{2} d B_{2}(t) .
$$

It then follows

$$
\begin{aligned}
\log \Phi_{2}(t)= & \log \Phi_{2}(0)-r_{2} t \\
& +b_{21} \int_{0}^{t} \Phi_{1}(s) d s-b_{22} \int_{0}^{t} \Phi_{2}(s) d s-\sigma_{2} B_{2}(t),
\end{aligned}
$$

With Lemma 6 and Assumption 8, we could get

$$
\begin{aligned}
\lim _{t \rightarrow \infty} \frac{1}{t} \int_{0}^{t} \Phi_{2}(s) d s \\
\quad=\frac{-r_{2}+b_{21}\left(r_{1} / b_{11}\right)}{b_{22}}=\frac{r_{1} b_{21}-r_{2} b_{11}}{b_{11} b_{22}}>0 .
\end{aligned}
$$

Let (38) divide $t$, and $t \rightarrow \infty$, together with (36) and (39), consequently

$$
\lim _{t \rightarrow \infty} \frac{\log \Phi_{2}(t)}{t}=0
$$

Similarly, according to Itô's formula, the third population of system (25) is changed into

$$
d \log \Phi_{3}(t)=\left(-r_{3}+b_{32} \Phi_{2}(t)-b_{33} \Phi_{3}(t)\right) d t-\sigma_{3} d B_{3}(t) ;
$$

it then follows

$$
\begin{gathered}
\log \Phi_{3}(t)=\log \Phi_{3}(0)-r_{3} t+b_{32} \int_{0}^{t} \Phi_{2}(s) d s \\
-b_{33} \int_{0}^{t} \Phi_{3}(s) d s-\sigma_{3} B_{3}(t), \\
\lim _{t \rightarrow \infty} \frac{1}{t} \int_{0}^{t} \Phi_{3}(s) d s \\
=\frac{-r_{3}+b_{32}\left(\left(r_{1} b_{21}-r_{2} b_{11}\right) / b_{11} b_{22}\right)}{b_{33}}>0, \\
\lim _{t \rightarrow \infty} \frac{\log \Phi_{3}(t)}{t}=0 .
\end{gathered}
$$

From this, together with Lemmas 7 and 9, the following result is obviously true.

Theorem 10. If Assumption 8 is satisfied, the solution $x(t)$ of system (3) with any initial value $x(0) \in R_{+}^{3}$ has the following property:

$$
\limsup _{t \rightarrow \infty} \frac{\log x_{i}(t)}{t} \leq 0, \quad i=1,2,3 .
$$

Above all, we could get.

Theorem 11. If Assumption 8 is satisfied, the the solution $x(t)$ of system (3) with any initial value $x(0) \in R_{+}^{3}$ has the following property:

$$
\liminf _{t \rightarrow \infty} \frac{1}{t} \int_{0}^{t} x_{3}(s) d s \geq \tilde{x}_{3}^{*}, \text { a.s., }
$$

where $\tilde{x}^{*}=\left(\tilde{x}_{1}^{*}, \tilde{x}_{2}^{*}, \tilde{x}_{3}^{*}\right)$ is the only nonnegative solution of the following equation:

$$
\begin{gathered}
r_{1}-b_{11} x_{1}-b_{12} x_{2}=0, \\
-r_{2}+b_{21} x_{1}-b_{22} x_{2}-b_{23} x_{3}=0, \\
-r_{3}+b_{32} x_{2}-b_{33} x_{3}=0 .
\end{gathered}
$$


Proof. From system (3), such that

$$
\begin{aligned}
& d\left(c_{1} \log x_{1}(t)+c_{2} \log x_{2}(t)+c_{3} \log x_{3}(t)\right) \\
& =\left[\left(r_{1} c_{1}-r_{2} c_{2}-r_{3} c_{3}\right)+\left(-b_{11} c_{1}+b_{21} c_{2}\right) x_{1}\right. \\
& \quad+\left(-b_{12} c_{1}-b_{22} c_{2}+b_{32} c_{3}\right) x_{2} \\
& \left.\quad-\left(b_{23} c_{2}+b_{33} c_{3}\right) x_{3}\right] d t \\
& +c_{1} \sigma_{1} d B_{1}(t)-c_{2} \sigma_{2} d B_{2}(t)-c_{3} \sigma_{3} d B_{3}(t) .
\end{aligned}
$$

Let $c_{1}=b_{21}, c_{2}=b_{11}$, and $c_{3}=\left(b_{11} b_{22}+b_{12} b_{21}\right) / b_{32}$, together with Assumption 8, we know

$$
r_{1} c_{1}-r_{2} c_{2}-r_{3} c_{3}>0
$$

hence

$$
\begin{aligned}
\left(c_{1}\left(\log x_{1}(t)-\log x_{1}(0)\right)+c_{2}\left(\log x_{2}(t)-\log x_{2}(0)\right)\right. & \\
& \left.+c_{3}\left(\log x_{3}(t)-\log x_{3}(0)\right)\right) \times(t)^{-1} \\
= & \left(r_{1} c_{1}-r_{2} c_{2}-r_{3} c_{3}\right) \\
& -\left(c_{2} b_{23}+c_{3} b_{33}\right) \frac{1}{t} \int_{0}^{t} x_{3}(s) d s \\
+ & \frac{c_{1} \sigma_{1} B_{1}(t)-c_{2} \sigma_{2} B_{2}(t)-c_{3} \sigma_{3} B_{3}(t)}{t} .
\end{aligned}
$$

According to Theorem 10, where

$$
\limsup _{t \rightarrow \infty} \frac{\log x_{i}(t)}{t} \leq 0, \quad i=1,2,3,
$$

and $\lim _{t \rightarrow \infty}\left(B_{i}(t) / t\right)=0, i=1,2,3$,

$$
\liminf _{t \rightarrow \infty} \frac{1}{t} \int_{0}^{t} x_{3}(s) d s \geq \frac{r_{1} c_{1}-r_{2} c_{2}-r_{3} c_{3}}{c_{2} b_{23}+c_{3} b_{33}}=\tilde{x}_{3}^{*},
$$

where $\tilde{x}^{*}=\left(\tilde{x}_{1}^{*}, \tilde{x}_{2}^{*}, \tilde{x}_{3}^{*}\right)$ is the only nonnegative solution of the following equation when Assumption 8 is satisfied:

$$
\begin{gathered}
r_{1}-b_{11} x_{1}-b_{12} x_{2}=0, \\
-r_{2}+b_{21} x_{1}-b_{22} x_{2}-b_{23} x_{3}=0, \\
-r_{3}+b_{32} x_{2}-b_{33} x_{3}=0 .
\end{gathered}
$$

\section{Nonpersistence}

In this section, we show the situation when the population of system (3) will be extinction in three cases.

Case $1\left(r_{1}<0\right)$. According to Itô's formula, the first population of system (25) is changed into

$$
d \log \Phi_{1}(t) \leq\left(r_{1}-b_{11} \Phi_{1}(t)\right) d t-\sigma_{1} d B_{1}(t) .
$$

If $r_{1}<0$, we could get

$$
\limsup _{t \rightarrow \infty} \frac{\log \Phi_{1}(t)}{t} \leq \frac{r_{1}}{b_{11}}<0 \text { a.s. }
$$

From the stochastic comparison theorem, we have

$$
\limsup _{t \rightarrow \infty} \frac{\log x_{1}(t)}{t} \leq \frac{r_{1}}{b_{11}}<0 \text { a.s., }
$$

hence

$$
\lim _{t \rightarrow \infty} x_{1}(t)=0, \text { a.s. }
$$

From the second population of system (25), we have

$$
\begin{aligned}
& \limsup _{t \rightarrow \infty} \frac{\log \Phi_{2}(t)}{t} \\
& \quad \leq-a_{2}+b_{21} \limsup _{t \rightarrow \infty} \frac{1}{t} \int_{0}^{t} \Phi_{1}(s) d s \leq-a_{2} \text { a.s.; }
\end{aligned}
$$

similarly

$$
\begin{aligned}
& \limsup _{t \rightarrow \infty} \frac{\log \Phi_{3}(t)}{t} \leq-a_{3} \text { a.s., } \\
& \lim _{t \rightarrow \infty} x_{i}(t)=0 \quad \text { a.s. } \mathrm{i}=2,3 .
\end{aligned}
$$

Case $2\left(r_{1}>0, r_{1}-\left(b_{11} / b_{21}\right) r_{2}<0\right)$. It is clear that from the proof section of Case 1 , we get

$$
\begin{aligned}
& \frac{\log \Phi_{2}(t)-\log \Phi_{2}(0)}{t} \\
& \quad \leq-r_{2}+b_{21} \frac{1}{t} \int_{0}^{t} \Phi_{1}(s) d s-\frac{\sigma_{2} d B_{2}(t)}{t} \text { a.s., }
\end{aligned}
$$

hence

$$
\begin{aligned}
& \limsup _{t \rightarrow \infty} \frac{\log \Phi_{2}(t)}{t} \\
& \leq-r_{2}+b_{21} M_{1}=-r_{2}+b_{21} \frac{r_{1}}{b_{11}}<0 \text { a.s. }
\end{aligned}
$$

Similarly

$$
\begin{aligned}
& \limsup _{t \rightarrow \infty} \frac{\log \Phi_{3}(t)}{t} \\
& \quad \leq-r_{3}+b_{32} \limsup _{t \rightarrow \infty} \frac{1}{t} \int_{0}^{t} \Phi_{2}(s) d s \\
& \quad \leq-a_{3}<0 \text { a.s.; }
\end{aligned}
$$

thus,

$$
\lim _{t \rightarrow \infty} x_{i}(t)=0 \quad \text { a.s., } \mathrm{i}=2,3 .
$$

Above all, and from the conclusion in [22], we could easily know that the distribution of $x_{1}(t)$ converges weekly to the probability measure with density:

$$
f^{*}(\zeta)=C_{0} \zeta^{2 r_{1} / \sigma_{1}^{2}-1} e^{-2 b_{11} \zeta / \sigma_{1}^{2}}
$$

where $C_{0}=\left(2 b_{11} / \sigma_{1}^{2}\right)^{2 r_{1} / \sigma_{1}^{2}} / \Gamma\left(2 r_{1} / \sigma_{1}^{2}\right)$ and

$$
\lim _{t \rightarrow \infty} \frac{1}{t} \int_{0}^{t} x_{1}(s) d s=\frac{r_{1}}{b_{11}}, \text { a.s. }
$$


Case $3\left(r_{1}-\left(b_{11} / b_{21}\right) r_{2}-\left(\left(b_{11} b_{22}+b_{12} b_{21}\right) / b_{21} b_{32}\right) r_{3}<0\right)$. It is clear that

$$
\begin{aligned}
d\left(c_{1} \log x_{1}(t)+c_{2} \log x_{2}(t)+c_{3} \log x_{3}(t)\right) \\
=\left[\left(r_{1} c_{1}-r_{2} c_{2}-r_{3} c_{3}\right)+\left(-b_{11} c_{1}+b_{21} c_{2}\right) x_{1}\right. \\
\quad+\left(-b_{12} c_{1}-b_{22} c_{2}+b_{32} c_{3}\right) x_{2} \\
\left.\quad-\left(b_{23} c_{2}+b_{33} c_{3}\right) x_{3}\right] d t \\
+c_{1} \sigma_{1} d B_{1}(t)-c_{2} \sigma_{2} d B_{2}(t)-c_{3} \sigma_{3} d B_{3}(t) .
\end{aligned}
$$

Since $c_{1}=b_{21}, c_{2}=b_{11}, c_{3}=\left(b_{11} b_{22}+b_{12} b_{21}\right) / b_{32}$, we get

$$
\begin{aligned}
& c_{1} \log x_{1}(t)+c_{2} \log x_{2}(t)+c_{3} \log x_{3}(t) \\
& \leq\left(r_{1} c_{1}-r_{2} c_{2}-r_{3} c_{3}\right) t \\
&+c_{1} \log x_{1}(0)+c_{2} \log x_{2}(0)+c_{3} \log x_{3}(0) \\
&+c_{1} \sigma_{1} B_{1}(t)-c_{2} \sigma_{2} B_{2}(t)-c_{3} \sigma_{3} B_{3}(t),
\end{aligned}
$$

Moreover,

$$
\begin{aligned}
& \frac{\log x_{1}^{c_{1}}(t) x_{2}^{c_{2}}(t) x_{3}^{c_{3}}(t)}{t} \\
& \leq\left(r_{1} c_{1}-r_{2} c_{2}-r_{3} c_{3}\right) \\
& \quad+\frac{c_{1} \log x_{1}(0)+c_{2} \log x_{2}(0)+c_{3} \log x_{3}(0)}{t} \\
& \quad+c_{1} \sigma_{1} \frac{B_{1}(t)}{t}-c_{2} \sigma_{2} \frac{B_{2}(t)}{t}-c_{3} \sigma_{3} \frac{B_{3}(t)}{t} .
\end{aligned}
$$

And $\lim _{t \rightarrow \infty}\left(B_{i}(t) / t\right)=0, i=1,2,3$, implies

$$
\limsup _{t \rightarrow \infty} \frac{\log x_{1}^{c_{1}}(t) x_{2}^{c_{2}}(t) x_{3}^{c_{3}}(t)}{t} \leq r_{1} c_{1}-r_{2} c_{2}-r_{3} c_{3}<0
$$

then

$$
\lim _{t \rightarrow \infty} x_{1}^{c_{1}}(t) x_{2}^{c_{2}}(t) x_{3}^{c_{3}}(t)=0 \text { a.s. }
$$

Therefore, by the above arguments, we get the following conclusion.

Theorem 12. Let $x(t)$ be the solution of system (3) with any initial value $x(0) \in R_{+}^{3}$. Then

(1) if $r_{1}<0$, then

$$
\lim _{t \rightarrow \infty} x_{i}(t)=0 \quad \text { a.s., } \mathrm{i}=1,2,3,
$$

(2) if $r_{1}>0, r_{1}-\left(b_{11} / b_{21}\right) r_{2}<0$, then

$$
\lim _{t \rightarrow \infty} x_{i}(t)=0 \quad \text { a.s., } \mathrm{i}=2,3 \text {, }
$$

and the distribution of $x_{1}(t)$ converges weekly to the probability measure with density:

$$
f^{*}(\zeta)=C_{0} \zeta^{2 r_{1} / \sigma_{1}^{2}-1} e^{-2 b_{11} \zeta / \sigma_{1}^{2}}
$$

where $C_{0}=\left(2 b_{11} / \sigma_{1}^{2}\right)^{2 r_{1} / \sigma_{1}^{2}} / \Gamma\left(2 r_{1} / \sigma_{1}^{2}\right)$ and

$$
\lim _{t \rightarrow \infty} \frac{1}{t} \int_{0}^{t} x_{1}(s) d s=\frac{r_{1}}{b_{11}}, \text { a.s; }
$$

(3) if $r_{1}-\left(b_{11} / b_{21}\right) r_{2}-\left(\left(b_{11} b_{22}+b_{12} b_{21}\right) / b_{21} b_{32}\right) r_{3}<0$, then

$$
\lim _{t \rightarrow \infty} x_{1}^{c_{1}}(t) x_{2}^{c_{2}}(t) x_{3}^{c_{3}}(t)=0 \text {, a.s., }
$$

where $c_{1}=b_{21}, c_{2}=b_{11}$, and $c_{3}=\left(b_{11} b_{22}+b_{12} b_{21}\right) / b_{32}$.

\section{Numerical Simulation}

In this section, we give out the numerical experiment to support our results. Consider

$$
\begin{aligned}
\dot{x}_{1}(t)= & x_{1}(t)\left(a_{1}-b_{11} x_{1}(t)-b_{12} x_{2}(t)\right) \\
& +\sigma_{1} x_{1}(t) \dot{B}_{1}(t), \\
\dot{x}_{2}(t)= & x_{2}(t)\left(-a_{2}+b_{21} x_{1}(t)-b_{22} x_{2}(t)-b_{23} x_{3}(t)\right) \\
& -\sigma_{2} x_{2}(t) \dot{B}_{2}(t) \\
\dot{x}_{3}(t)= & x_{3}(t)\left(-a_{3}+b_{32} x_{2}(t)-b_{33} x_{3}(t)\right) \\
& -\sigma_{3} x_{3}(t) \dot{B}_{3}(t) .
\end{aligned}
$$

By the Milstein method in [23], we have the difference equation:

$$
\begin{aligned}
x_{1, k+1}= & x_{1, k}+x_{1, k} \\
\times & {\left[\left(a_{1}-b_{11} x_{1, k}-b_{12} x_{2, k}\right) \Delta t\right.} \\
& \left.+\sigma_{1} \epsilon_{1, k} \sqrt{\Delta} t+\frac{\sigma_{1}^{2}}{2}\left(\epsilon_{1, k}^{2} \Delta t-\Delta t\right)\right], \\
x_{2, k+1}= & x_{2, k}+x_{2, k} \\
\times & {\left[\left(-a_{2}+b_{21} x_{1, k}-b_{22} x_{2, k}-b_{23} x_{3, k}\right) \Delta t\right.} \\
& \left.-\sigma_{2} \epsilon_{2, k} \sqrt{\Delta} t+\frac{\sigma_{2}^{2}}{2}\left(\epsilon_{2, k}^{2} \Delta t-\Delta t\right)\right], \\
x_{3, k+1}=x_{3, k} & +x_{3, k} \\
\times & {\left[\begin{array}{l}
\left(-a_{3}+b_{32} x_{2, k}-b_{33} x_{3, k}\right) \Delta t \\
\quad
\end{array}\right.} \\
& \left.-\sigma_{3} \epsilon_{3, k} \sqrt{\Delta} t+\frac{\sigma_{3}^{2}}{2}\left(\epsilon_{3, k}^{2} \Delta t-\Delta t\right)\right],
\end{aligned}
$$

where $\epsilon_{1, k}, \epsilon_{2, k}$, and $\epsilon_{3, k}, i=1,2,3$, are the Gaussian random variables $N(0,1), r_{1}=a_{1}-\sigma_{1}^{2} / 2>0$, and $r_{i}=a_{i}+\sigma_{i}^{2} / 2, i=2,3$. Choosing $\left(x_{1}(0), x_{2}(0), x_{3}(0)\right) \in R_{+}^{3}$, and suitable parameters, by Matlab, we get Figures 1,2 , and 3 . 

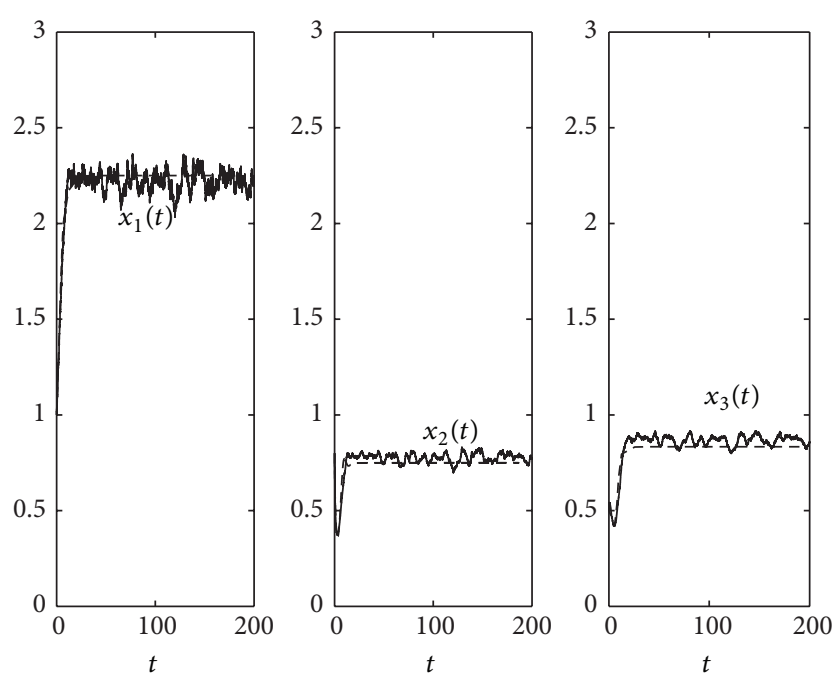

FIgURE 1: The solution of system (1) and system (3) with $\left(x_{1}(0), x_{2}(0), x_{3}(0)\right)=(1,0.8,0.5), a_{1}=0.3, a_{2}=0.4, a_{3}=0.1$, $b_{11}=0.1, b_{12}=0.1, b_{21}=0.6, b_{22}=0.6, b_{23}=0.6, b_{32}=0.8$, and $b_{33}=0.6$. The red lines represent the solution of system (1), while the blue lines represent the solution of system (3) with $\sigma_{1}=0.02$, $\sigma_{2}=0.01$, and $\sigma_{3}=0.01$.
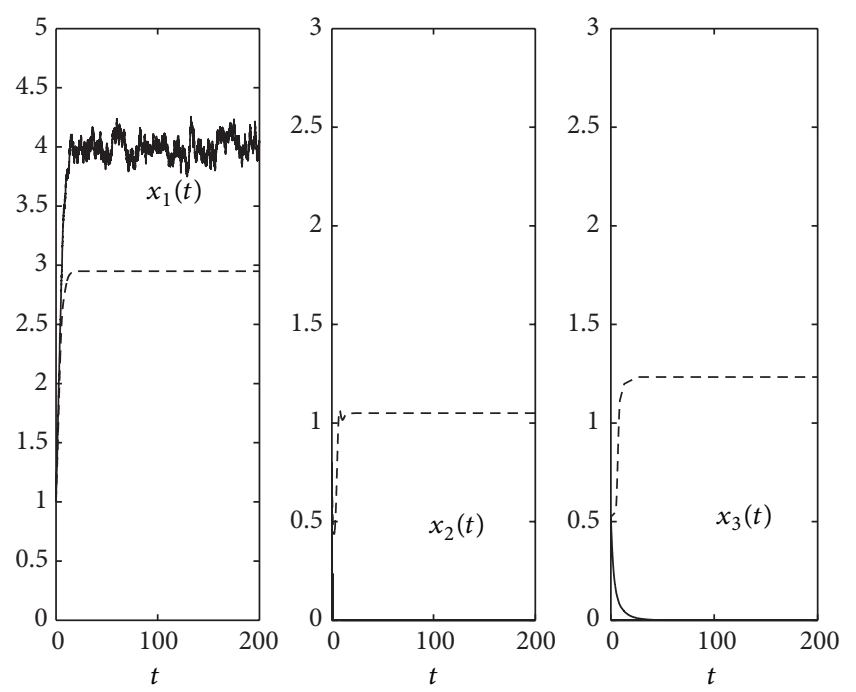

FIgURE 2: Two of the species will die out in probability. The red lines represent the solution of system (1), while the blue lines represent the solution of system (3) with $\left(x_{1}(0), x_{2}(0), x_{3}(0)\right)=(1,0.8,0.5)$, $a_{1}=0.4, a_{2}=0.4, a_{3}=0.1, b_{11}=0.1, b_{12}=0.1, b_{21}=0.6, b_{22}=0.6$, $b_{23}=0.6, b_{32}=0.8$, and $b_{33}=0.6$. The red lines represent the solution of system (1), while the blue lines represent the solution of system (3) with $\sigma_{1}=0.02, \sigma_{2}=3$, and $\sigma_{3}=0.01$.

In Figure 1, when the noise is small, choosing parameters satisfying the condition of Theorem 10 , the solution of system (3) will persist in time average.

In Figure 2, we observe case (3) in Theorem 12 and choose parameters $r_{1}>0, r_{1}-\left(b_{11} / b_{21}\right) r_{2}<0$. As Theorem 12 indicated that two predators will die out in probability. The prey solution of system (3) will persist in time average.
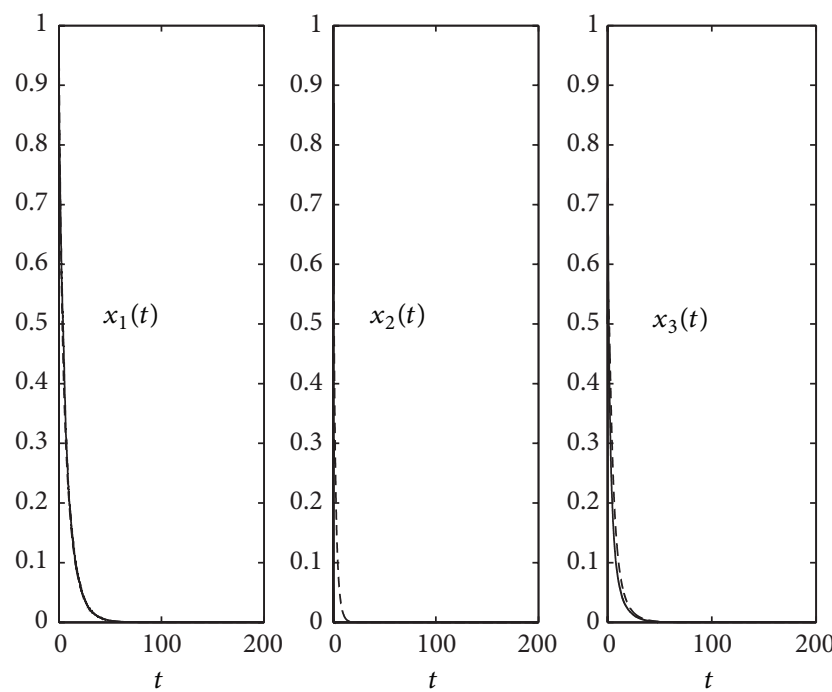

FIGURE 3: One of the species or both species will die out in probability. The red lines represent the solution of system (1), while the blue lines represent the solution of system (3) with $\left(x_{1}(0), x_{2}(0), x_{3}(0)\right)=(1,0.8,0.5), a_{1}=-0.1, a_{2}=0.4, a_{3}=0.1$, $b_{11}=0.1, b_{12}=0.1, b_{21}=0.6, b_{22}=0.6, b_{23}=0.6, b_{32}=0.8$, and $b_{33}=0.6$. The red lines represent the solution of system (1), while the blue lines represent the solution of system (3) with $\sigma_{1}=0.02$, $\sigma_{2}=3$, and $\sigma_{3}=0.01$.

In Figure 3, we observe case (1) in Theorem 12 and choose parameters $r_{1}<0$. As Theorem 12 indicated that not only predators but also prey will die out in probability when the noise of the prey is large, and it does not happen in the deterministic system.

\section{Acknowledgments}

The work was supported by the Ministry of Education of China (no. 109051), the Ph.D. Programs Foundation of Ministry of China (no. 200918), and NSFC of China (no. 11371085), Natural Science Foundation of Jilin Province of China (no. 201115133).

\section{References}

[1] B. S. Goh, "Global stability in many species system," American Naturalist, vol. 111, pp. 135-143, 1997.

[2] H. I. Freedman, Deterministic Mathematical Models in Population Ecology, vol. 57, Marcel Dekker, New York, NY, USA, 1980.

[3] Y. Kuang and H. L. Smith, "Global stability for infinite delay Lotka-Volterra type systems," Journal of Differential Equations, vol. 103, no. 2, pp. 221-246, 1993.

[4] K. Gopalsamy, "Global asymptotic stability in a periodic LotkaVolterra system," Journal of Australian Mathematical Society B, vol. 24, pp. 160-170, 1985.

[5] T. C. Gard, Introduction to Stochastic Differential Equations, vol. 114, Marcel Dekker, New York, NY, USA, 1988.

[6] T. C. Gard, "Persistence in stochastic food web models," Bulletin of Mathematical Biology, vol. 46, no. 3, pp. 357-370, 1984. 
[7] T. C. Gard, "Stability for multispecies population models in random environments," Nonlinear Analysis. Theory, Methods \& Applications, vol. 10, no. 12, pp. 1411-1419, 1986.

[8] X. Mao, G. Marion, and E. Renshaw, "Environmental Brownian noise suppresses explosions in population dynamics," Stochastic Processes and Their Applications, vol. 97, no. 1, pp. 95-110, 2002.

[9] X. Mao, "Delay population dynamics and environmental noise," Stochastics and Dynamics, vol. 5, no. 2, pp. 149-162, 2005.

[10] Y. Hu, F. Wu, and C. Huang, "Stochastic Lotka-Volterra models with multiple delays," Journal of Mathematical Analysis and Applications, vol. 375, no. 1, pp. 42-57, 2011.

[11] N. I. Wantanabe, Stochastic Differential Equations and Diffusion Processes, North-Holland, Amsterdam, The Netherlands, 1981.

[12] C. Ji, D. Jiang, N. Shi, and D. O'Regan, "Existence, uniqueness, stochastic persistence and global stability of positive solutions of the logistic equation with random perturbation," Mathematical Methods in the Applied Sciences, vol. 30, no. 1, pp. 77-89, 2007.

[13] C. Zhu and G. Yin, "On competitive Lotka-Volterra model in random environments," Journal of Mathematical Analysis and Applications, vol. 357, no. 1, pp. 154-170, 2009.

[14] C. Ji, D. Jiang, and N. Shi, "Analysis of a predator-prey model with modified Leslie-Gower and Holling-type II schemes with stochastic perturbation," Journal of Mathematical Analysis and Applications, vol. 359, no. 2, pp. 482-498, 2009.

[15] C. Ji, D. Jiang, and X. Li, "Qualitative analysis of a stochastic ratio-dependent predator-prey system," Journal of Computational and Applied Mathematics, vol. 235, no. 5, pp. 1326-1341, 2011.

[16] R. M. May, Stability and Complexity in Model Ecosystem, Princeton University Press, Princeton, NJ, USA, 2001.

[17] P. Polansky, "Invariant distributions for multipopulation models in random environments," Theoretical Population Biology, vol. 16, no. 1, pp. 25-34, 1979.

[18] M. Barra, D. G. Grosso, A. Gerardi, G. Koch, and F. Marchetti, Some Basic Properties of Stochastic Population Models, Springer, Berlin, Germany, 1979.

[19] X. R. Mao, Stochastic Differential Equations and Applications, Horwood, Chichester, UK, 1997.

[20] L. S. Chen and J. Chen, Nonlinear Biological Dynamical System, Science Press, Beijing, USA, 1993.

[21] P. Y. Xia, X. K. Zheng, and D. Q. Jiang, "Persistence and nonpersistence of a nonautonomous stochastic mutualism system," Abstract and Applied Analysis, vol. 2013, Article ID 256249, 13 pages, 2013.

[22] C. Y. Ji and D. Q. Jiang, "Dynamics of a stochastic density dependent predator-prey system with Beddington-DeAngelis functional response," Journal of Mathematical Analysis and Applications, vol. 381, no. 1, pp. 441-453, 2011.

[23] D. J. Higham, "An algorithmic introduction to numerical simulation of stochastic differential equations," SIAM Review, vol. 43 , no. 3 , pp. 525-546, 2001. 


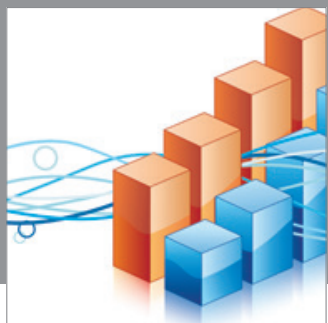

Advances in

Operations Research

mansans

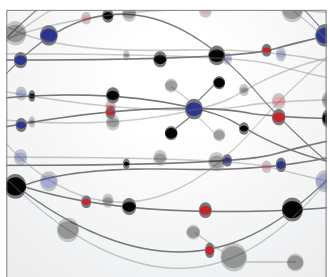

The Scientific World Journal
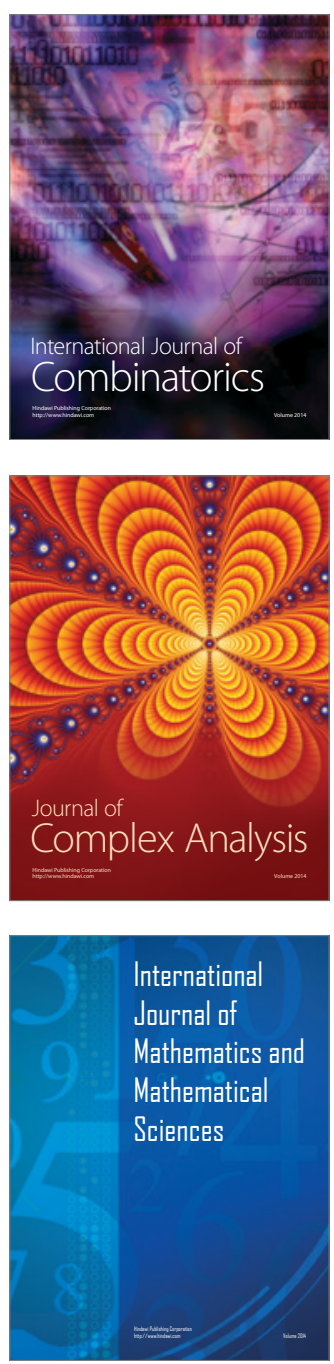
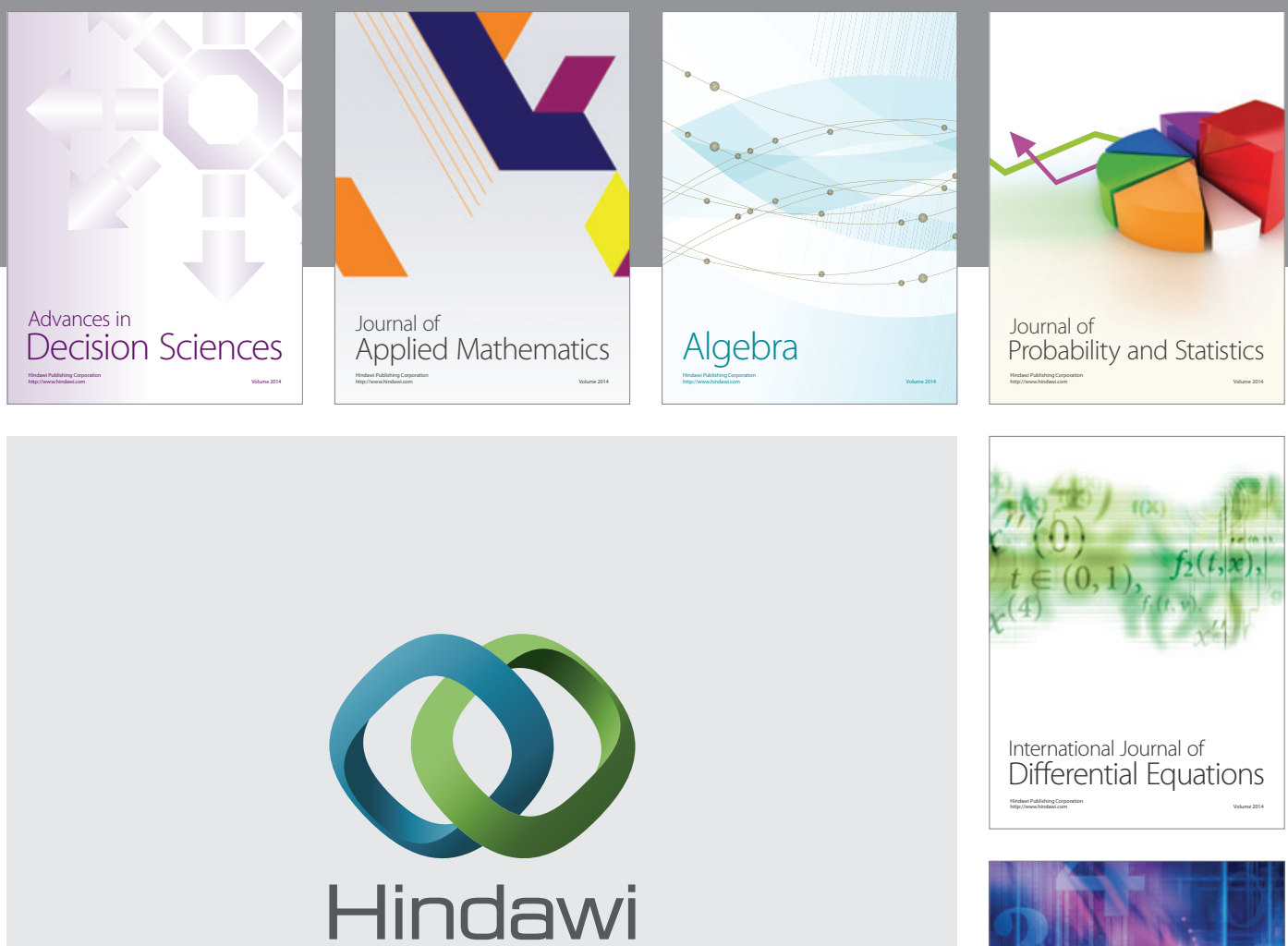

Submit your manuscripts at http://www.hindawi.com
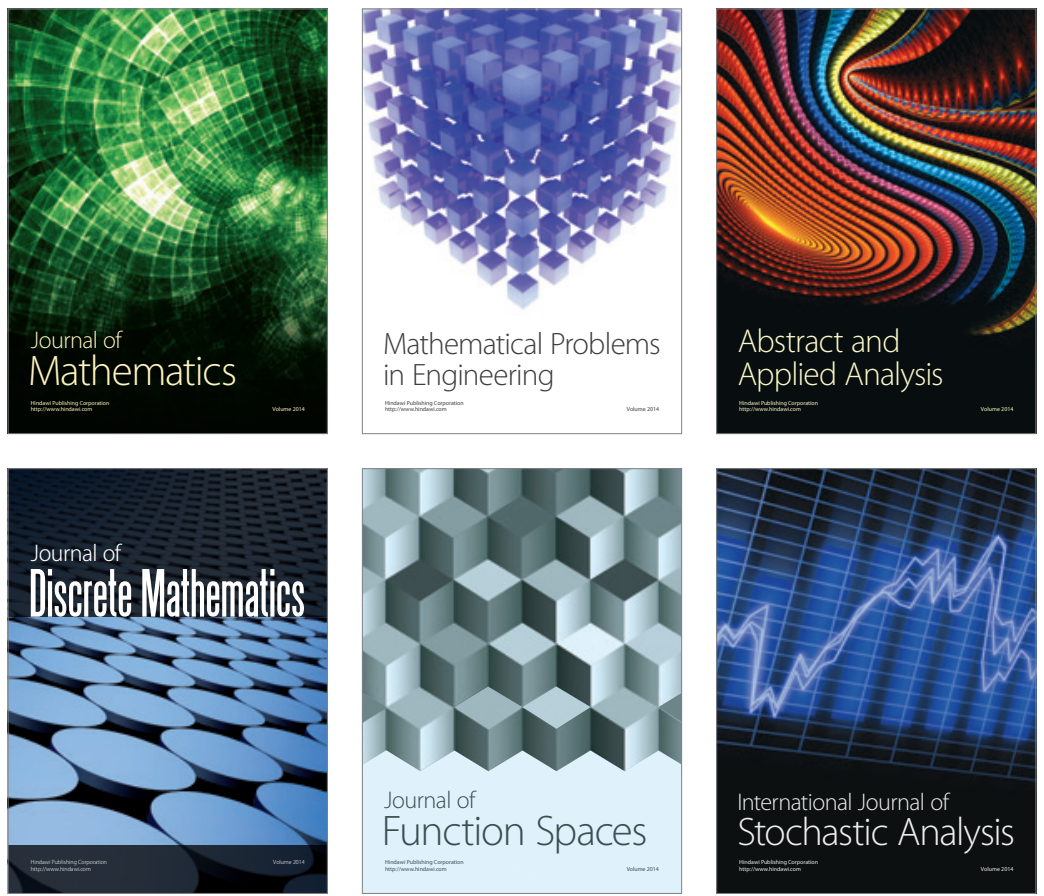

Journal of

Function Spaces

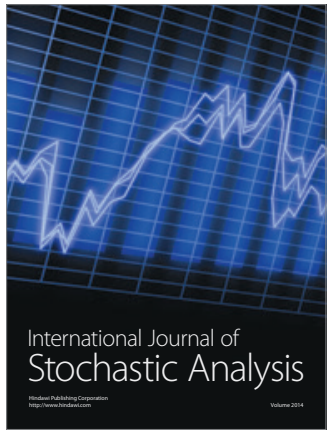

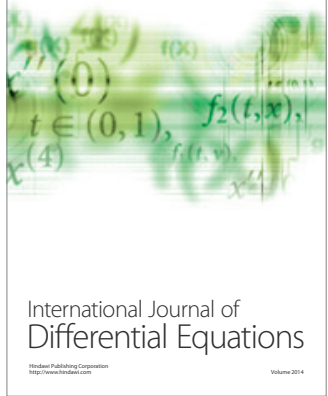
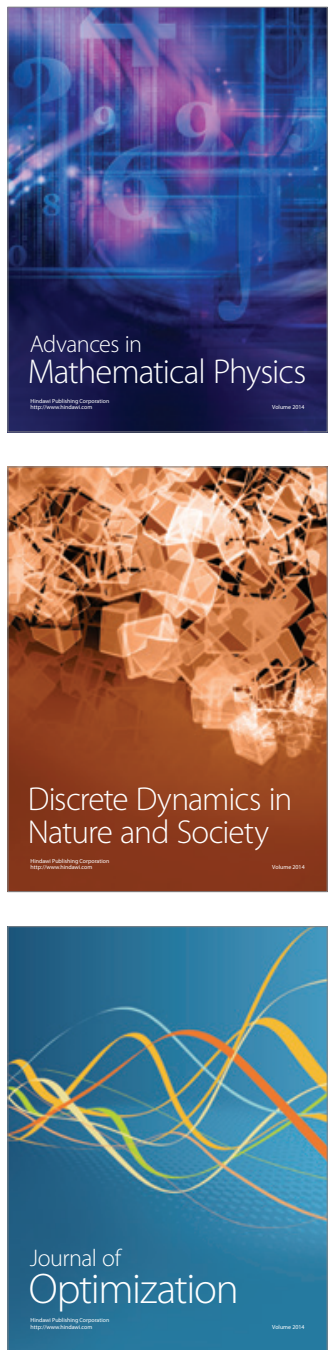